\title{
Channel-hole Based Scheduling in Relay-assisted Wireless Networks
}

\author{
Yan $\mathrm{Li}^{*}$, Yacine Ghamri-Doudane ${ }^{* \dagger}$ and Marwen Abdennebi* \\ ${ }^{*}$ ENSIIE, 1 Square de la résistance, 91025 Evry CEDEX, France \\ ${ }^{\dagger}$ LIGM - Université de Paris-Est Marne-la-Valle, 75420 Champs sur Marne, France \\ Email: \{li, ghamri, abdennebi\}@ensiie.fr
}

\begin{abstract}
Relay technologies are capable to significantly improve network performances in terms of cell capacity, coverage extension and system throughput. However, it should rely on an efficient scheduling scheme to fully profit from this potential gain. Although opportunistic scheduling is considered as the most promising scheduling technology for future networks in many literatures, it is not appropriate for the relay-assisted wireless systems due to some special features of the relay mode. In addition, most of the current literatures only discuss the scheduling in Base Station (BS) and neglect the channel state asymmetry between the BS-RS (Relay Station) link and RS-MS (Mobile Station) link. So we argue that an appropriate scheduling in RS can extremely improve the performance of the system. In this paper, we define a new concept called "channel-hole" which is used to precisely measure the wasted subchannel resources caused by the channel state asymmetry, and then propose a new scheduling algorithm, namely channel-hole based scheduling, in the RS based on this new concept. We also propose a theoretical model to analyze the proposed scheduling. Simulation results show that our proposed channel-hole scheduling is capable to utilize the radio resource more efficiently compared to the case where an opportunistic scheduling is used at the RS.
\end{abstract}

\section{INTRODUCTION}

Recently, relay technologies have been actively studied and considered in the standardization process of next-generation mobile broadband communication systems, such as IEEE 802.16j [1], and IEEE 802.16m [2]. The profit of introducing Relay Stations (RS) in the system is the extension of signal and service coverage, the enhancement of the system's overall throughput performance, and the power saving compared to the sole use of Base Stations (BS). The RS can forward high data rates in remote areas while keeping a low cost of infrastructure. But this potential gain in capacity and coverage is highly dependent on the scheduling scheme, a topic which draws more and more attention of the research community [3][4][5][6][7]. Some works on scheduling schemes can be found in [5][6][7], but they only focus on the scheduling in BS and assume that all the packets transmitted from the BS to RS are immediately relayed to the destination MS without the need of scheduling at the RS. However, as the channel states of the links (BS-RS and RS-MS) vary in time independently to each other, if there is asymmetry between the channel states of the BS-RS link and RS-MS link, radio resources might be wasted. This asymmetry also leads to potential queuing in the RS and thus to the necessity of scheduling. In [3], the authors studied the above mentioned problem and propose the use of opportunistic scheduling in both BS and RS. The basic idea is to allocate subchannels to the Mobile Station (MS) experiencing the best channel condition at each frame. However, as we will show throughout the paper, due to some specific features of relay networks, the opportunistic scheduling scheme is not the best choice for relay-assisted networks, although it is considered as the most promising scheduling algorithm in the non relay-assisted networks. For example, in a two-hop transmission, even the RS-MS link is good enough to be chosen according to opportunistic scheduling, it might be wasted if there is no buffered packets to be transmitted. Similarly, for the bad RS-MS link, the packets buffered in RS might be finally dropped after a long period as this bad RS-MS link will not be allocated with subchannels due to the principle of opportunistic scheduling. In this case, the BS-RS link capacity which is used to transmit these dropped packets is also wasted as the packets did not finally reached their destinations. Considering this as well as other situations that may arise while opportunistic scheduling is used at the RS level, we think that this kind of scheduling is not effective in relay based networks and thus not appropriate.

Actually, we strong believe that if the RS adequately allocates subchannels to those MSs with suboptimal RS-MS links status but who has queued packets in the RS buffer or those MSs with suboptimal BS-MS links but with good RS-MS links, the radio resource can be utilized more effectively. [4] notices this problem, but it assumes the RS-MS links are stable for long period of times (several frames) once their status are detected to be good. This assumption is not reliable and not precise as the channel status change frequently especially when the MS is moving with high speed or the communication environment is complete, such as in presence of massive high buildings. Hence, by using a the scheduling scheme based on such assumption, it is still possible to result in radio resource wasting due to link asymmetry.

Considering the above mentioned issues, we will first define in this paper a new concept, channel-hole, as the measurement for the wasted radio resource in the following frames when the channel varies. Then, in an aim to efficiently use the radio resources, we propose a new scheduling algorithm to be used in the RS based on this new concept. Since the radio resource waste situation (i.e. channel-hole) is more precisely defined than in [4] in asymmetric links, the proposed scheduling algorithm is expected to be more efficient and 


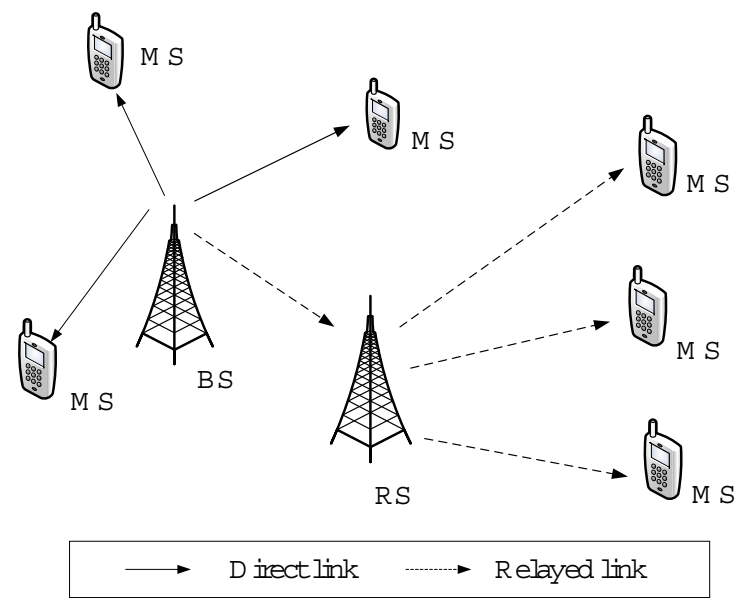

Fig. 1: Relay model.

improve the system performance more significantly than when using opportunistic scheduling in the RS. We also validate this by simulations.

The remainder of this paper is organized as follows. The system model and the problem statement are presented in Section II. The term channel-hole is also defined in this section. In Section III, we propose the channel-hole based scheduling algorithm followed in Section IV by the link capacity analysis needed by our algorithm. The simulation results are provided in Section V. We conclude the paper and give directions for future work in Section VI.

\section{System Model and Problem Statement}

\section{A. System Model}

In this paper, we consider a single cell downlink transmission using Orthogonal Frequency-Division Multiple Access (OFDMA), with one BS and one RS in the cell as depicted in Figure 1. The frame structure is illustrated in Figure 2. In the frequency domain, the downlink channel is divided into $N$ orthogonal subchannels each of which is constituted of a group of adjacent subcarriers. In the time domain, each frame is divided into a BS subframe and an RS subframe. In the BS subframe, only the BS can transmit data to the RS and MSs while in the RS subframe, the BS and RS can transmit data to MSs. Hence, an RSs can receive data only in the BS subframe and transmit data only in the RS subframe, while the BS can transmit data in both subframes. It is also assumed that the RS cannot transmit and receive data simultaneously.

Moreover, we assume the wireless channel is time-varying and frequency-selective but it is supposed to be flat within a subchannel and to be unchanged during a frame period. So Adaptive Modulation and Coding (AMC) will perform on per frame basis, and MSs feed back to the BS or to the RS their Channel State Information (CSI) containing their persubchannel Signal-to-Noise-Ratio (SNR) in each frame.

Then, similarly to [3], we define the following sets of elements:

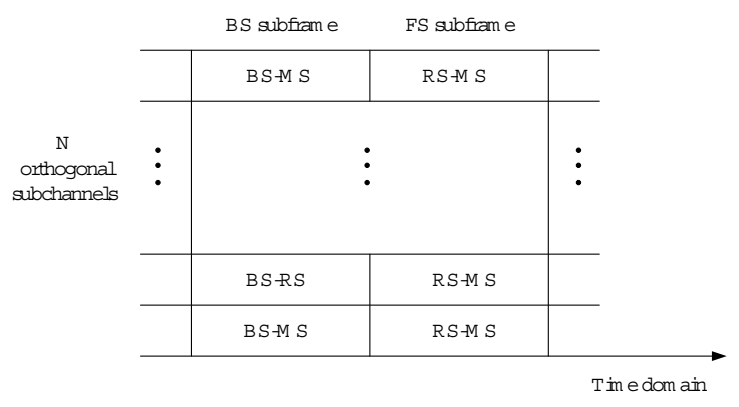

Fig. 2: Frame structure for the relay-assisted networks.

$$
\begin{array}{ll}
\mathcal{K}=\left\{k_{0}, k_{1}\right\} & \text { representing the BS and RS; } \\
\mathcal{M}=\{1,2, \cdots, M\} & \text { representing the MSs; } \\
\mathcal{N}=\{1,2, \cdots, N\} & \text { representing the subchunnels; } \\
\mathcal{T}=\{1,2\} & \text { representing the subframes. }
\end{array}
$$

For $\mathcal{K}, " k_{0}$ " represents the BS and " $k_{1}$ "represents the RS. For $\mathcal{T}$, "1" represents the BS subframe and " 2 " represents the RS subframe. During a frame, all the data are delivered to the destination (RS in the BS subframe and MS in the RS subframe) through a logical link $(s, d)$, where $s \in \mathcal{K}$ and $d \in k_{1} \cup \mathcal{M}$. Different combinations of $s$ and $d$ constitute the aggregate $\mathcal{X}=\left\{x_{0}, x_{1}, \cdots, x_{i}, \cdots\right\}$, i.e. $(s, d) \in \mathcal{X}$.

Since each subchannel is assigned to each subframe, there is no intracell interference. During the $t-t h$ frame, we define the subchannel assignment indicator:

$$
d_{t}(l, n, \tau)= \begin{cases}1 & \text { if subchannel } n \text { is assigned to the } \\ & l-t h \text { path at subframe } \tau \\ 0 & \text { otherwise }\end{cases}
$$

where $n \in N, l \in \mathcal{X}$ and $\tau \in \mathcal{T}$.

We suppose the power is equally distributed on each subchannel which gives:

$$
P_{\tau}(n)=\frac{P_{t o t}}{N}
$$

where $P_{t o t}$ is the total transmission power of the system.

The noise in the system is defined as

$$
\sigma^{2}=N_{0} \frac{B}{N}
$$

where $B$ is the bandwidth and $N_{0}$ is the power spectrum density of the noise.

We also define the subchannel gain of the $l-t h$ link on the subchannel $n$ as $h_{t}(l, n, \tau)$, where $n \in \mathcal{N}, l \in \mathcal{X}, \tau \in \mathcal{T}$. So during the $t-t h$ frame, the instantaneous capacity of the $l-t h$ link on the subchannel $n$ can be written as:

$$
c_{t}(l, n, \tau)=\frac{B}{N} \log _{2}\left(1+\frac{p_{\tau}(n)\left|h_{t}(l, n, \tau)\right|^{2}}{\sigma^{2}}\right)
$$

Then, during the $t-t h$ frame, the capacity of the $l-t h$ link can be calculated as:

$$
R_{t}(l)=\sum_{n=1}^{N} \sum_{\tau=1}^{2} d_{t}(l, n, \tau) c_{t}(l, n, \tau)
$$




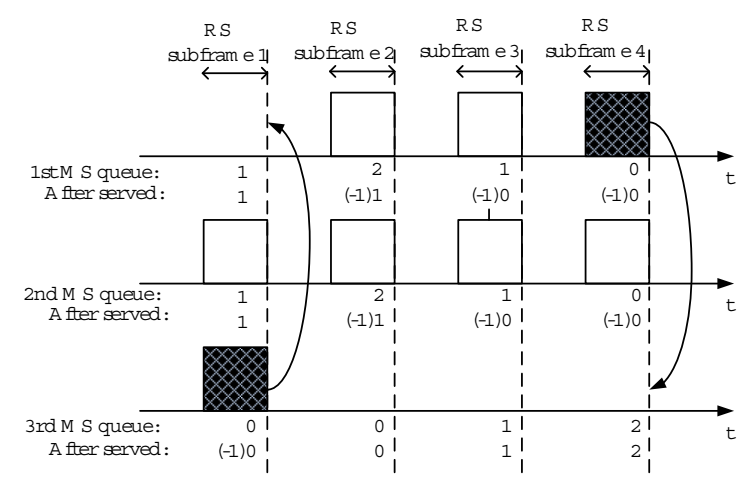

Fig. 3: Example of the "holes" in subchannels.

\section{B. Problem statement}

As mentioned in the introduction section, opportunistic scheduling is not efficient in relay-assisted systems due to the specificities of the relay feature. Here we will present a simple example, illustrated in Figure 3, to explain with more details the "channel-hole" problem and then show how our proposed scheduling scheme will deal with this problem.

For simplicity and visibility, in Figure 3, it is temporarily assumed that there are only three MSs in the system, and two subchannels are allocated to the RS-MS link in the four frames shown in the Figure 3, i.e. from $3^{\text {rd }}$ frame to $4^{\text {th }}$ frame. Here we mainly illustrate the "hole" in subchannels during the RS subframe because the RS-MS link is the major bottleneck in the relay transmission. But the same problem can also happen in the BS subframe. We assume that there are three different small buffers in the RS buffer storing the packets to be forwarded to the corresponding destination MSs. Since only two subchannels are available, only two RS-MS links have right to forward packets in each RS frame, as denoted by squares in the figure. We list 3 statistics in the example, which are newly arrived packets through BS-RS link and to be stored in the RS buffer, i.e. "new arrival", number of queued packets sorted by the specific MS destination in the RS buffer when the RS subframe begins, i.e. "queue length", and the packet number that the RS served, i.e. "after served". "queue length" correspond to the sum of "new arrival" in current RS subframe and the "after served" in the previous RS subframe. "after served" is calculated by the "queue length" minus the number of packets that are forwarded to the destination in the same frame.

When the opportunistic scheduling is adopted in RS, it always chooses the two RS-MS links which have the best CSI to forward the packets. The squares in the figure represents the chosen link. For example, in the $1^{\text {st }} \mathrm{RS}$ subframe, the squares show the $2^{\text {nd }}$ RS-MS link and the $3^{\text {rd }}$ RS-MS link has better link condition than others. So, the packets buffered in the RS queue, which are destined to the $2^{\text {nd }}$ MS and the $3^{\text {rd }}$ MS, can be forwarded during this RS subframe. We also simply assume only one packet can be forwarded for each chosen link in a RS subframe. Actually, in the real system, the number of packets which can be transmitted are decided by the AMC and is also different in each frame. In the $1_{s t}$ subframe, "after served" equals to the "queue length" because its RS-MS link is not chosen; packets in the RS buffer and that are destinated to $2^{\text {nd }}$ MS is zero after one packet is forwarded; $3^{\text {rd }}$ "after served" equals to " 1 " because there is no queued packets whose destination is $3^{\text {rd }}$ MS. But $3^{\text {rd }}$ RS-MS link is allocated with subchannel according to our definition of "after served". So, $3^{\text {rd }}$ "after served" should then be "-1" as the $3^{\text {rd }}$ MS queue is 0 .

We notice that in the $1^{\text {st }}$ RS subframe, the radio resource which is allocated to the $3^{\text {rd }}$ RS-MS link is wasted because there is no packet that needs to be forwarded although this link has better CSI than the $1^{\text {st }}$ link. We use the term "channel hole" to define this situation, which is defined as the number of packets which are supposed to be forwarded but actually not due to the fact that RS buffer is empty. Hence, in the example above, the channel-holes related to the $3^{\text {rd }}$ RS-MS link should be "1". It is notable that channel-holes is also linked to the instantaneous channel rate, that is if the channel-hole problem is expected happen, high channel rates means a high number of channel-holes.

However, in the $1^{\text {st }}$ RS subframe, although the link condition of $1^{\text {st }}$ RS-MS link is worse than the $3^{\text {rd }}$ RS-MS link, there is queued packets for the destination of $1^{s t}$ MS. So we can imagine to assign the subchannel to the $1^{\text {st }}$ RS-MS link. In this case, the radio resource will not be completely wasted. Indeed, even if with bad channel condition, the $1^{\text {st }}$ RS-MS can still forward some packets to the destination even the number of these packets is less than the one that can go through the $3^{\text {rd }}$ RS-MS when there are buffered packets to the $3^{\text {rd }}$ MS. So, it can be supposed that if these channel-holes can be used, the system performance will be improved by reducing the wasted channel capacity.

\section{ChANNEL-HOLE BASED SChEdULING ALGORITHM}

Based on the above analysis, we propose in this section an improved scheduling algorithm which is called channelhole based scheduling. In this paper, we assume there exist a fixed or dynamic path selection that decides whether the MSs should select the relayed link or the direct link. Once the path is selected for each MS, the selected links are kept until the long-term average SNR of the MS change significantly. The path selection is performed periodically by assessing the long-term channel statistics. The proposed scheduling scheme is described in Algorithm 1.

As mentioned in section II, we assume that the channel rate to be flat within a frame and unchanged during it. So in this algorithm, we first estimate the instantaneous capacities and sort them in descending order. The sorting list contains BS-MS links, BS-RS links and RS-MS links. As we mainly consider the scheduling scheme in RS, the scheduling in BS can implement any. Here we use the opportunistic scheduling in the BS subframe, i.e. the BS always chooses the best links for its transmissions, which includes BS-MS direct transmissions and BS-RS relayed transmission. Each subchannel is assigned to only one link in each subframe. Actually, the allocations 


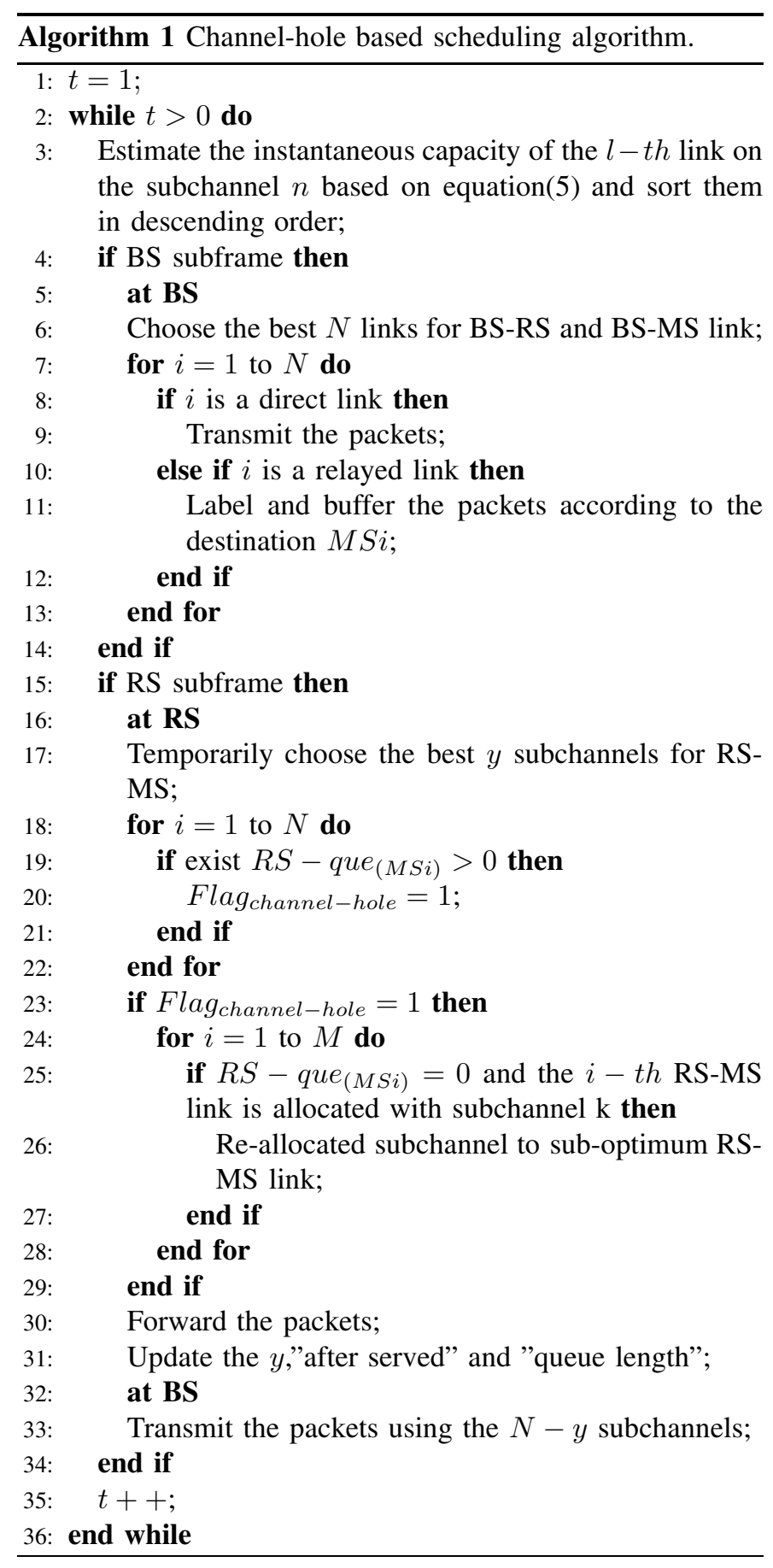

are also performed according to the path selection which determines the number of direct links and relayed links in each frame. Our focus is to improve the performance of relay links. Thus here we simply first satisfy the direct links and then use the left subchannels to support the relay transmissions.

In the BS subframe, the packets sent from the BS and buffered in the RS will constitute the RS queue and they are classified and accumulated according to the destination MS, that is $R S-q u e_{(M S i)}$, while $i=1,2, \cdots, M$. Then in the $\mathrm{RS}$ subframe, the direct link is given the higher priority and will be allocated $N-y$ subchannels while the remaining $y$ subchannels are used for the RS-MS links. The RS starts by temporarily allocating the subchannel to the top $y$ RS-MS links which have good link statuses. Then, the RS check whether this allocation leads to channel-hole problems. The principle is if the $i-t h$ RS-MS link is allocated with subchannels by the RS and the $i-t h$ MS queue length is zero in the meanwhile, there will be a channel-hole. It is notable that if all the $R S-q u e_{(M S i)}$ equals to zero, it means no packets in the RS queue. In this case, even it belongs to the channel-hole concept according to the definition, it is actually not because there are no packets in the buffer and no more subchannel become needed. We use Flag channel-hole $=1$ to denote the existence of a channel hole. When Flag channel-hole $=1$, it means that a channel-hole problem is experienced and that re-allocation is needed. When Flag channel-hole equals to 1 , the $\mathrm{RS}$ choose the sub-optimized RS-MS links to use the channel-hole resources. The RS check the sorting list in the $M-y$ remaining links and find the links whose $\mathrm{RS}$ queue length is bigger than zero, which means the links that have packets in the buffer and needs suchannel resources to forward them. Our scheduling algorithm will thus re-allocate the subchannel to them. After the transmission, the "after served" and "queue length" parameters need to be updated for each MS, since these variables are used for channel-hole detection. One should also note that the "after served" value should be set to zero here when the channel-hole problem happens. One should finally note that when no channel-hole is experienced (Flag channel-hole $=0$ ) after the first allocation to the RS-MS links, the subchannel suplus is given to the direct BS-MS lnks.

\section{THEORETICAL ANALYSIS}

In this section, we discuss the theoretical analysis of the proposed channel-hole scheduling algorithm. More precisely, we aim in this section at deriving the exact capacity expression which is needed in our scheduling algorithm to sort the links according to their capacities. We first define the average arrival rate and average service rate for each $R S-q u e_{(M S i)}=k$ as bing $\lambda_{i, k}$ and $\mu_{i, k}$ respectively with $i=1,2, \cdots, M$. For different MSs, the $R S-q u e_{(M S i)}$ is stochastic. There are four possible statuses for each $R S-q u e_{(M S i)}$ during a small time period $(t, t+h)$ when $h \rightarrow 0$ :

1) 0 packets arrive and 0 packets are forwarded with probability $\left(1-\lambda_{i, k} h\right)\left(1-\mu_{i, k} h\right)$;

2) 0 packets arrive and 1 packet is forwarded with probability $\left(1-\lambda_{i, k+1} h\right) \mu_{i, k+1} h$;

3) 1 packet arrives and 0 packets are forwarded with probability $\lambda_{i, k-1} h\left(1-\mu_{i, k-1} h\right)$;

4) 1 packet arrives and 1 packet is forwarded with probability $\lambda_{i, k} h \cdot \mu_{i, k} h$;

So, the transition probability at $t+h$ for the $i-t h$ MS with $k$ packets in the queue length can be written as: 


$$
\begin{aligned}
P_{i, k}(t+h)= & P_{i, k}(t)\left(1-\lambda_{i, k} h\right)\left(1-\mu_{i, k} h\right) \\
& +P_{i, k+1}(t)\left(1-\lambda_{i, k+1} h\right) \mu_{i, k+1} h \\
& +P_{i, k-1}(t) \lambda_{i, k-1} h\left(1-\mu_{i, k-1} h\right) \\
& +P_{i, k}(t) \lambda_{i, k} h \cdot \mu_{i, k} h
\end{aligned}
$$

where $t>0, k>0, i=1,2, \cdots, M$, and $h$ is infinitesimal of higher order.

Thus, we define the following equation which represents the differential equation for the transition probability when $t$ tends to 0 .

$$
\begin{aligned}
\frac{d P_{i, k}(t)}{d t} & =\lim _{h \rightarrow 0} \frac{P_{i, k}(t+h)-P_{i, k}(t)}{h} \\
& =\left\{\begin{array}{l}
-P_{i, 0}(t) \lambda_{0}+P_{i, 1}(t) \mu_{1}, \quad k=0 \\
P_{i, n-1}(t) \lambda_{i, n-1} h \\
-P_{i, n}(t)\left(\lambda_{i, n}+\mu_{i, n}\right) \\
+P_{i, n+1}(t) \mu_{i, n+1} h,
\end{array} \quad k \neq 0 .\right.
\end{aligned}
$$

If the system is stable, the transition probability matrix $\Lambda$ of the system should satisfy $\mathrm{P} \Lambda=0$, where $\mathrm{P}=$ $\left[\pi_{i, 0}, \pi_{i, 1}, \cdots, \pi_{i, k}, \cdots \pi_{i, K}\right]$ is the probability distribution vector for each $R S-q u e_{(M S i)}$ and $\pi_{i, k}$ is the value of stable probability for $P_{i, k}$ when $k=1,2, \cdots, K ; K>0$; $i=1,2, \cdots, M$. With equation (7), we can calculate $\mathrm{P}$ as follows:

$$
\begin{array}{ll}
\lambda_{i, o} \pi_{i, 0}=\mu_{i, 1} \pi_{i, 1}, & k=0 \\
\left(\lambda_{i, k}+\mu_{i, k}\right) \pi_{i, k}=\lambda_{i-1, k} \pi_{i-1, k}+\mu_{i+1, k} \pi_{i+1, k}, & k>0
\end{array}
$$

where $i=1,2, \cdots, M$. In the mean while, above equation also satisfies:

$$
\sum_{k=0}^{\infty} \pi_{i, k}=1, i=1,2, \cdots, M .
$$

Combining (8) and (9), the elements of stable probability distribution vector are calculated as:

$$
\begin{gathered}
\pi_{i, 0}=\left(1+\sum_{k=1}^{\infty} \frac{\lambda_{i, 0} \lambda_{i, 1} \cdots \lambda_{i, k-1}}{\mu_{i, 1} \mu_{i, 2} \cdots \mu_{i, k}}\right)^{-1} \\
\pi_{i, k}=\frac{\lambda_{i, 0} \lambda_{i, 1} \cdots \lambda_{i, k-1}}{\mu_{i, 1} \mu_{i, 2} \cdots \mu_{i, k}}\left(1+\sum_{j=1}^{\infty} \frac{\lambda_{i, 0} \lambda_{i, 1} \cdots \lambda_{i, k-1}}{\mu_{i, 1} \mu_{i, 2} \cdots \mu_{i, k}}\right)^{-1} \\
k>0, i=1,2, \cdots, M
\end{gathered}
$$

As each subchannel has the same opportunity to be used for the service because they have no priority for each other, the subchannel capacity can be considered to obey the same probability distribution, i.e. $\mu_{1, j}=\mu_{2, j}=\cdots=\mu_{M, j}=\mu$. Then equation (10) can be expressed by:

$$
\pi_{i, k}=\frac{\left(\lambda_{i, k} / \mu\right)^{k}}{1+\sum_{k=1}^{\infty}\left(b \lambda_{i, k} / \mu\right)^{k}}
$$

As the subchannel assignment indicator $d_{t}(l, n, \tau)$, obey $(0,1)$ the distribution, the probability that $d_{t}(l, n, \tau)$ equals
TABLE I: Simulation Settings

\begin{tabular}{|c|c|}
\hline Parameter & Value \\
\hline \hline Number of BS & 1 \\
\hline Number of MSs & 15 \\
\hline Number of subchannels & 128 \\
\hline Frame lenghth & $10 \mathrm{~ms}$ \\
\hline Path loss exponents for BS-RS links & 2 \\
\hline Standard deviation of shadow fading & 4 \\
\hline Power of BS & $8 \mathrm{~dB}$ \\
\hline Power of RS & $40 \mathrm{w}$ \\
\hline
\end{tabular}

to 1 is the probability to have $\left.R S_{(q u e}(M S i)\right)>0$ according to our algorithm, that is

$$
P\left(d_{t}(l, n, \tau)=1\right)=\sum_{i=1}^{M}\left(1-\frac{1}{1-\lambda_{i, 0} / \mu}\right) .
$$

With equation (5) and (12), the capacity of the $l-t h$ link during the $\tau-t h$ frame can be expressed by:

$$
\begin{gathered}
R_{t}(l)=\sum_{n=1}^{N} \sum_{\tau=1}^{2} \sum_{i=1}^{M}\left(1-\frac{1}{1-\lambda_{i, 0} / \mu}\right) c_{t}(l, n, \tau) \\
\text { V. Simulation RESULTS }
\end{gathered}
$$

In this section, we present the simulation results for the proposed scheduling algorithm. Parameter settings of the simulation are shown in Table 1. The simulations are made in a single cell with a radius of $1000 \mathrm{~m}$, containing one BS and one RS. The RS is placed $800 \mathrm{~m}$ away from the BS. MSs are distributed uniformly in the cell. The time-varying frenquency-selective channels are modeled by using pathloss, shadow fading and fast Rayleigh fading [8]. We assume that the channel condition of each subcarrier varies independently with each other. We assume that if the distance between the BS and an MS is smaller than $800 \mathrm{~m}$, all subchannels of the MS are allocated to the one-hop path in both the BS subframe and the RS subframe. Otherwise, these subchannels are allocated to the two-hop path through the RS. Simulations are made for a duration of 10000 frames. In the simulation, we compare the performance when opportunistic scheduling and our proposed channel-hole based scheduling are adopted in the RS. In the BS, we use the opportunistic scheduling, that is the BS allocates the subchannel to the link which have the best CSI.

Figure 4 compares the queue length in the RS buffer when different scheduling algorithm are adopted. The packet arrival processes for all the MSs follow the Poisson distribution with arrival rate of 0.3 packets/frame. Figure 4(a) shows the RS queue length when the opportunistic scheduling is used in the RS, while Figure 4(b) shows the RS queue length when our proposed channel-hole based scheduling is used in the RS. As we can see in Figure 4(a), the RS queue length increases more dramatically than that in Figure 4(b). This means that the opportunistic scheduling cannot well deal with the buffered packets in such situation and these packets cannot be sent 


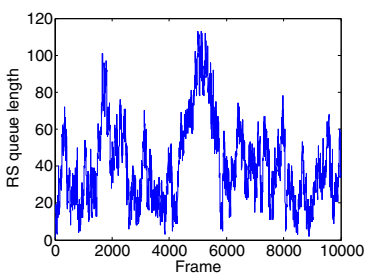

(a)

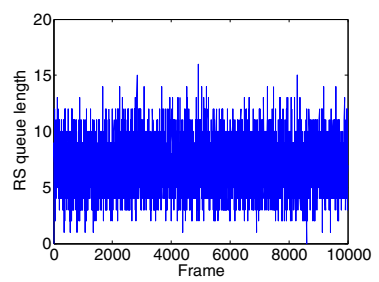

(b)
Fig. 4: RS queue length with (a) opportunistic schduling and (b)channel-hole based scheduling in the RS.

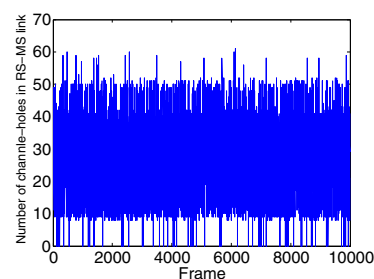

(a)

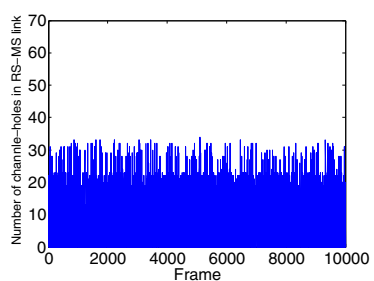

(b)
Fig. 5: Number of Channel-holes with (a) opportunistic schduling and (b)channel-hole based scheduling in the RS.

out in time leading to congestion. Since we do not consider the packet loss rate caused by long delays, it is possible to record the packets number in the buffer. Contrarily, from Figure 4(b), we can see that the queue length in RS can keep a small value, i.e. a maximum of 15 , which is clearly less than that in Figure 4(a). Thus we denotes that with our proposed scheduling algorithm, the resources can be used more efficiently to forward the buffered packets through the relay link, which results in small queue lengths.

Figure 5 compares the number of channel-holes in RSMS link as defined in the Section II when both scheduling schemes are used. The packet arrival processes for all the MSs also follow the Poisson distribution with arrival rate of 0.3 packets/frame. Figure 5(a) shows the number of channel-holes when the opportunistic scheduling algorithm is used in the RS and Figure 5(b) shows the number of channel-holes when our proposed scheduling is used in the RS. With this figure, we can easily see that the number of channel-holes in Figure 5(a) is higher than that in Figure 5(b). Actually, channel-holes indicate the wasted subchannels. The wasted radio resource when opportunistic scheduling is used are twice more than wasted when our channel-hole based scheduling is used in the RS.

Finally, Figure 6 shows the channel utilization varying with the arrival rate of the MSs when opportunistic scheduling and our proposed channel-hole scheduling are adopted respctively. Again, the packet arrival processes follow the Poisson distribution. From Figure.8, we can see when the arrival rates increase, the channel utilization also increase. However, the channel-hole resource scheduling gives better results than the opportunistic scheduling and keeps higher

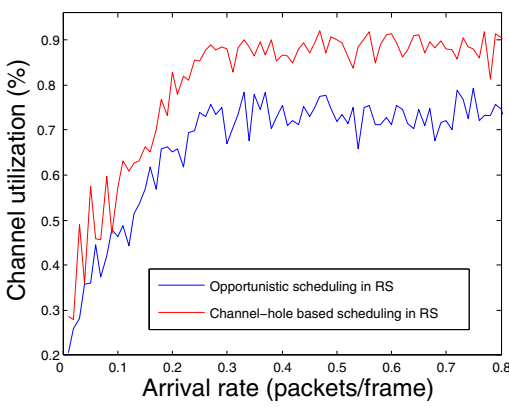

Fig. 6: Channel utilization when different scheduling algorithms are used in the RS.

utilization percentage because the former try to use the links which are not the best but with queued packets to be sent.

\section{CONCLUSION}

In this paper, we first discussed the special features of relayassisted network, and disclosed the shortcomings of opportunistic scheduling when it is used in RSs for relay-assisted wireless networks. According to this, we define a new concept, i.e. channel-hole, which is linked to the wasted subchannel resources caused by link asymmetry. This concept precisely measures the wasted subchannel resources. To efficiently use the subchannel resources especially when asymmetric links exists, we proposed a new scheduling algorithm which is called channel-hole based scheduling to be used in the RS. For the purpose of our scheduling algorithm, we also derived analytically the link capacity expression. Simulations showed that by allowing the proposed scheduling at the RS, the amount of wasted subchannels are significantly lower to those wasted while adapting the opportunistic scheduling at RS. In the mean while, the system performance in terms of resource utilization and buffer occupancy are also improved. In our future work, we aim to study the case where multiple RSs are used and extend our scheduling to handle this case.

\section{ACKNOWLEDGMENT}

This work is supported by European project ITEA2 HDTVnext.

\section{REFERENCES}

[1] IEEE P802.16j/D9, "Draft Amendment to IEEE Standard for Local and Metropolitan Area Networks Part 16: Air Interface for Fixed and Mobile Broadband Wireless Access Systems: Multihop Relay Specification,' Feb. 2009.

[2] WiMAX Forum, "Network Architecture Stage 2-3," Rel.1, version 1.2, Jan.2008, http://www.wimaxforum.org/technology/documents

[3] K. Byung-Gook and L. Jang-Won, "Opportunistic Power Scheduling for OFDMA Cellular Networks with Scheduling at Relay Stations," IEEE WCNC 2009, pp. 1-6.

[4] M. Kaneko and P. Popovski, "Radio Resource Allocation Algorithm for Relay-Aided Cellular OFDMA System," IEEE ICC 2007, pp. 4831-4836.

[5] H. Chi-Yao and P. Ai-Chun, "3-Approximation algorithm for joint routing and link scheduling in wireless relay networks," IEEE Transactions on Wireless Communications, vol. 8, pp. 856-861, 2009.

[6] P. Tarasak and S. Sumei, "Joint Cooperative Diversity and Proportional Fair Scheduling in OFDMA Relay Systems," IEEE VTC 2008-Fall, pp. 1-5.

[7] G. Senarath et al. "Multi-hop Relay System Evaluation Methodology," $80216 j-06_{0} 13 r 3$, July 2007 